\title{
Microarray Missing Data Imputation based on a Set Theoretic Framework and Biological Constraints
}

\author{
Xiangchao Gan ${ }^{1}$, Alan Wee-Chung Liew ${ }^{2}$ and Hong Yan ${ }^{1,3}$ \\ ${ }^{l}$ Department of Electronic Engineering \\ City University of Hong Kong, 83 Tat Chee Avenue, Kowloon, Hong Kong \\ ${ }^{2}$ Department of Computer Science and Engineering \\ The Chinese University of Hong Kong, Shatin, Hong Kong \\ ${ }^{3}$ School of Electrical and Information Engineering \\ University of Sydney, NSW 2006, Australia
}

\begin{abstract}
Gene expressions measured using microarrays usually suffer from the missing value problem. Existing missing value imputation algorithms have some limitations. For example, some algorithms have good performance only when strong local correlation exists in data while some provide the best estimate when data is dominated by a global structure. In addition, these algorithms do not take into account many biological constraints in the imputation procedure. In this paper, we propose a set theoretic framework for missing data imputation. We design our algorithm by taking into consideration the biological characteristic of the data and exploit the local correlation and the global correlation structure adaptively. Experiments show that our algorithm can achieve a significant reduction of error compared with existing methods.
\end{abstract}

\section{Introduction}

DNA Microarray has been widely used in numerous biological disciplines, such as the investigation of drug action and cancer prognosis. However, microarray data often contain missing values with measurements for many genes affected. Missing values occur due to various reasons, including hybridization failures, artifacts on the microarray, insufficient resolution and image noise.

There are several simple methods to deal with missing values [1]. Although all these algorithms have shown good performance to deal with missing values when the required condition is satisfied, they also have their limitations. KNNimpute performs better on nontime series data or noisy time series data, while SVDimpute works well on time series data with low noise level and with strong global correlation structure. BPCA is suitable when a global structure is dominant in data. Nevertheless, these algorithms do not consider biological constraints related to the microarray experiments.

In this paper, we propose a new missing value imputation algorithm that has a superior performance to existing algorithms. This is an extension of our earlier work [2] and the novelty of our current method in this paper lies in two aspects. First, our algorithm can exploit the local and global correlation structures in microarray data adaptively. Second, we make explicitly use synchronization loss, a biological phenomenon in microarray experiment, as a constraint in the imputation process.

\section{Biological property for missing values imputation}

In a microarray experiment, synchronization is achieved by first arresting cells at a specific biological life point and then releasing cells from the arrest so that all cells are at the same point when the experiment begins [3]. However, even if cells are synchronized perfectly at the beginning of the experiment, they do not remain synchronized forever [4]. For example, yeast cells seem to remain relatively synchronized for two cycles while wild type human cells lose their synchronization very early or halfway through the first cycle depending on the arresting method. This causes the peak expression value to be lower in the second cycle and the lowest expression value to be higher for most cycling genes. A typical gene expression profile with synchronization loss is given in Fig. 1. Due to this phenomenon, we find that the average signal power in successive cycles decreases significantly. Table 1 
shows our statistical results of four datasets in Spellman et al.'s experiment.

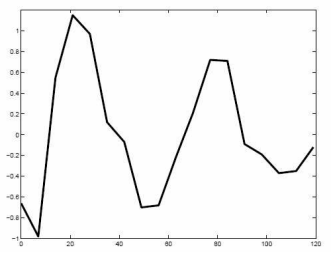

Fig. 1. The gene expression profile of Smc3 in Spellman et al.'s experiment. The synchronization loss is significant.

\begin{tabular}{|c|c|c|c|c|}
\hline Dataset & $\begin{array}{c}\text { No. of } \\
\text { sampling } \\
\text { points }\end{array}$ & $\begin{array}{c}\text { No. of } \\
\text { complete } \\
\text { genes }\end{array}$ & $\begin{array}{c}\text { Average } \\
\text { energy, } \\
\text { 1st cycle }\end{array}$ & $\begin{array}{c}\text { Average } \\
\text { energy, } \\
\text { 2nd cycle }\end{array}$ \\
\hline CDC28 & 17 & 1383 & 352.78 & 288.73 \\
\hline CDC15 & 24 & 4381 & 846.28 & 834.57 \\
\hline Alpha factor & 18 & 4489 & 474.58 & 306.24 \\
\hline Elutriation & 14 & 5766 & 898.48 & 435.32 \\
\hline
\end{tabular}

Table 1. The statistical result of synchronization loss for 4 datasets in Spellman et al.'s experiment. Note that since the signal of Elutriation is available only for one cycle, we compare the average signal energy for firsthalf cycle and the second-half cycle.

\section{The STF-based Imputation Algorithm}

The Set Theoretic Framework (STF) provides a convenient framework to allow multiple pieces of prior information of different nature to be utilized to get an optimal solution. It has been used successfully in signal and image processing [5, 6]. In this method, every known a priori property about the original signal is formulated as a corresponding convex set in a Hilbert space. Given $m$ closed convex sets $C_{i}, i=1,2, \cdots, m$, and nonempty intersection $C_{0}=\bigcap_{i=1}^{m} C_{i}$, the simultaneous projections onto the convex sets

$$
a_{n+1}=\sum_{l=1}^{m} w_{l} P_{l}\left(a_{n}\right)
$$

will converge to a point in the intersection $C_{0}$ for any initial $a_{0}$, where $a_{n}$ is the estimation of the signal at iteration $n$, and $P_{i}$ is the projector onto $C_{i}$ defined by

$$
\left\|\mathbf{x}-P_{i}(\mathbf{x})\right\|=\min _{\mathbf{g} \in C_{i}}\|\mathbf{x}-\mathbf{g}\|
$$

Another useful feature of the POCS algorithm is its adaptivity in finding a good solution. This can be explained as follow. Suppose we have correlation information between genes and between samples, and this two pieces of information are modeled as two convex sets $C_{u}$ and $C_{v}$, respectively. In one dataset, the first piece of information may be more reliable than the second. In another dataset, it may be the opposite. This situation is depicted in Fig. 2. When the information is more reliable, the corresponding convex set will be smaller in range. Since POCS always converge to the intersection, the final solution will always be dominated by the smaller set, while still satisfying the constraint imposed by the less reliable set. In this manner, a good solution that makes trade-off between different prior information can be obtained.

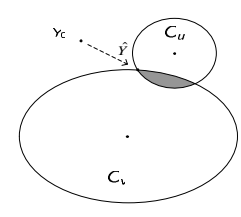

(a)

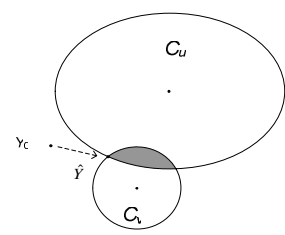

(b)
Fig. 2. POCS adaptivity: (a) In a data dominated by gene-wise correlation, the final solution is dominated by $C_{u}$, and (b) in a data dominated by array-wise correlation, the final solution is dominated by $C_{v}$.

\subsection{Capturing gene-wise correlation}

In gene expression data, genes that have close biological functions would express similarly. To capture this localized gene-wise correlation in the gene expression data, we construct a convex set based on local least square regression as in [7] as follows. First, we select the $K$-most correlated genes in $Y$ whose expression profile vectors are similar to gene $i$ except the $j$-th component and with the $j$-th component available. Then we estimate the missing value in the target gene using each reference gene based on the single regression model. If we denote the expression profile vector of the target gene as $\mathbf{y}$, for a reference gene $\mathbf{x}$, we have

$$
\mathbf{y}=\alpha+\beta \mathbf{x}+\mathbf{e}
$$

where e represents random noise. The parameters $\alpha$ and $\beta$ are estimated by

$$
\hat{\alpha}=\bar{y}-\hat{\beta} \bar{x} \text { and } \hat{\beta}=\frac{S_{x y}}{S_{x x}}
$$

where $S_{x y}=\frac{1}{n-1} \sum_{j=1}^{n}\left(x_{j}-\bar{x}\right)\left(y_{j}-\bar{y}\right)$, and $S_{x x}=\frac{1}{n-1} \sum_{j=1}^{n}\left(x_{j}-\bar{x}\right)^{2}$.

Thus the least squares estimate of a variable $f$ given a variable $t$ can be written as $\hat{f}=\bar{y}+\frac{S_{x y}}{S_{x x}}(t-\bar{x})$ and the variance of the residual error is given by

$$
\tau=\frac{1}{n-2} \sum_{i=1}^{n}\left(y_{i}-\hat{\alpha}-\hat{\beta} x_{i}\right)^{2}
$$


Since we have $K$ reference genes, we can obtain $K$ estimates $\hat{f}_{l}$ with variance of estimation error as $\tau_{l}(l=$ $1,2, \ldots, K)$ for a missing value. A weighted average of the estimates and the corresponding variance of the estimation error can be computed.

Defining the positions in matrix $Y$ of all missing values as a set $I$, we can get an estimate $\hat{B}(l), l \in I$ for every missing value using the above regression. Considering the possible estimation error in this method, we obtain a set

$$
C_{u}=\{Y: \hat{B}(l)-\tau \sqrt{p} \leq Y(l) \leq \hat{B}(l)+\tau \sqrt{p}, l \in I\}
$$

The projection $P_{u}$ onto convex set $C_{u}$ is then given by

$$
P_{u}(Y(l))=\left\{\begin{array}{cc}
\hat{B}(l)-\tau \sqrt{p} & \text { for } Y(l)<\hat{B}(l)-\tau \sqrt{p} \\
\hat{B}(l)+\tau \sqrt{p} & \text { for } Y(l)>\hat{B}(l)+\tau \sqrt{p} \\
Y(l) & \text { otherwise }
\end{array}\right.
$$

\subsection{Capturing array-wise correlation}

We use the PCA approach to capture the global array-wise variation. Assume we have a complete data matrix with no missing value. PCA represents the variation of each array vector $\mathbf{y}$ as a linear combination of principle axis vector $\boldsymbol{u}_{l}(0<l<\mathrm{K})$

$$
\mathbf{y}=\sum_{l=1}^{K} x_{l} \mathbf{u}_{l}+\boldsymbol{\varepsilon}
$$

The linear coefficients $x_{l}(0<l<K)$ are called factor scores and $\boldsymbol{\varepsilon}$ denotes the residual error. For each $\boldsymbol{u}_{l}$, there is a corresponding eigenvalue $\lambda_{l}$. For gene expression data, eigenvalue $\lambda_{l}$ indicates the relative significance of the $l$-th eigenarray in term of the fraction of the overall expression they captured. In PCA for gene expression data, only the $K(0<\mathrm{K}<\mathrm{L})$ most significant eigenarray are used [8]. The other $L-K$ eigenarray are treated as noise and the signal-to-noise ratio is given by

$$
p=\sum_{k=1}^{K} \lambda_{k}^{2} / \sum_{k=K+1}^{L} \lambda_{k}^{2}
$$

The estimation error is given by $\boldsymbol{\varepsilon}$. As in Equation (6), when a solution $\tilde{y}_{i, j}$ for a missing value is found using this method, a more reliable estimate is that the missing value lies in an interval $[(1-\tau \sqrt{p}) \tilde{y},(1+\tau \sqrt{p}) \tilde{y}]$, where $\tau$ is a parameter determined statistically. Defining the positions of all missing values in matrix $Y$ as a set $I$, we can construct a convex set

$$
C_{v}=\left\{Y: \varepsilon_{1} \tilde{A}(l) \leq Y(l) \leq \varepsilon_{2} \tilde{A}(l), l \in I\right\}
$$

where the $\widetilde{A}(l)$ is the estimated missing value using the eigenarrays, and $\varepsilon_{1}=(1-\tau \sqrt{p})$ and $\varepsilon_{2}=(1+\tau \sqrt{p})$. The projection onto set $C_{v}$ is then given by

$$
P_{v}(Y(l))=\left\{\begin{array}{cc}
\varepsilon_{1} \tilde{A}(l) & \text { for } Y(l)<\varepsilon_{1} \tilde{A}(l) \\
\varepsilon_{2} \tilde{A}(l) & \text { for } Y(l)>\varepsilon_{2} \tilde{A}(l) \\
Y(l) & \text { otherwise }
\end{array}\right.
$$

\subsection{Capturing the phenomenon of synchronization loss}

We propose here a series of convex sets to take advantage of the phenomenon of synchronization loss. Define the positions in matrix $Y$ of all missing values belonging to the $i$-th period as a set $I_{i}$, and the positions of all observed values belonging to the $i$-th period as a set $\Omega_{i}$. Let function $u(I)$ be the cardinal number of set $I$. We get

$$
C_{i}=\left\{Y: \quad \frac{1}{u\left(I_{i}\right)} \sum_{l \in I_{i}} Y^{2}(l)=\varphi_{i}\right\}
$$

with $\varphi_{i}=\frac{1}{u\left(\Omega_{i}\right)} \sum_{l \in \Omega_{i}} Y^{2}(l)$ and $i=1, \ldots, n$ denotes the number of periods considered.

The projection of an arbitrary $Y(i, j)$ onto the set $C_{i}$ is then given by

$$
P_{i}(Y(l))=\left\{\begin{array}{cc}
\frac{Y(l)}{\sqrt{\varphi_{i} \cdot u\left(I_{i}\right)}} & \text { for } l \in I_{i} \text { and } \sum_{l \in I_{i}} Y^{2}(l) \neq 0(13) \\
Y(l) & \text { otherwise }
\end{array}\right.
$$

\subsection{Our algorithm}

With the convex sets defined, the set theoretic estimation yields the following missing values imputation algorithm:

(1) Select average of the gene expression profile as initial estimation $Y_{0}$.

(2) For $k=1,2, \cdots$, compute $Y_{k}$ from

$Y_{k}=w_{1} P_{u}\left(Y_{k-1}\right)+w_{2} P_{v}\left(Y_{k-1}\right)+w_{3} P_{1} \cdots P_{n}\left(Y_{k-1}\right) \quad$ where $P_{u}, P_{v}, P_{1}, \cdots, P_{n}$ denote the projectors onto the constraint sets $C_{u}, C_{v}, C_{1} \cdots C_{n}$, respectively, and $w_{1}, w_{2}$ and $w_{3}$ are weighting parameters of POCS with $\sum_{l=1}^{3} w_{l}=1$ and $w_{l}>0$ for all $l=1,2,3$. A convenient choice is to let $w_{l}$ 's be equal.

(3) If $Y_{k}=Y_{k-1}$, exit the iteration, else go to step 2.

\section{Experiment results}

In this section, we apply our method to two microarray data sets. The first one is the study of yeast cell-cycle from Spellman et al. (http://cellcyclewww.stanford.edu) [3]. It contains expression profiles of 6178 genes under different experimental conditions, i.e., cdc15, and cdc28, alpha factor and elutriation experiments. In addition, one of the time-series data 
sets contained less apparent noise than the other. Another time series data set is from [8], which we denote as fkh1_fkh2.

The missing value estimation techniques were tested by randomly removing data values and then computing the estimation error. In the experiments, between $1-15 \%$ of the values is removed from each dataset. The normalized RMS (NRMS) error is calculated. We compare the normalized RMS error of our algorithm with the KNNimpute, SVDimpute and LSimpute algorithms. Due to limited space of the paper, we only provide an experiment with $15 \%$ missing values in Fig. 3.

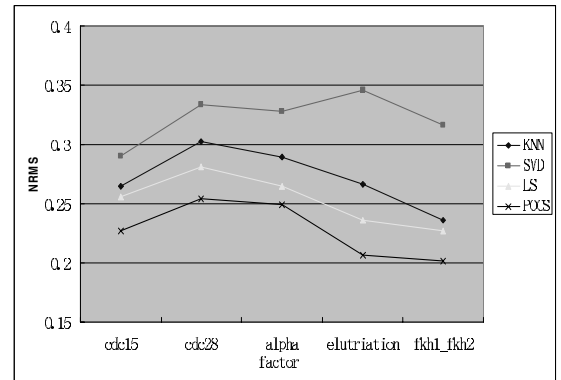

Fig. 3. Performance comparison for $15 \%$ missing values.

We now analyze the performance of the imputation algorithms as a function of gene expression level. The box and whisker plots of the estimation errors relative to the true expression values ( $\log 2$ ratio) in range are provided in Fig. 4. The test data are from Elutriation with 4489 genes and 18 arrays and $1 \%$ missing values. From the plots, we can find some interesting properties of our estimation. When the magnitude of true values is small, the performances of three methods are close. However our algorithm has lower error median and spread when true expression values are medium or large ( $\log 2$ ratio between 0.68 to 1.5 ). In a microarray experiment, the expression ratios of those genes with medium or high expression level are considered to be more reliable and hence taken with greater faith. If those values are missing due to experimental artifacts or contaminations, we would like them to be more reliably imputed. Our algorithm provides better estimate for these medium or large values.

\section{Conclusion}

In this paper, we have proposed a set theoretic approach based on POCS which we call POCSimpute for the problem of microarray missing value estimation. POCSimpute can adaptively find an optimal solution regardless of whether the global or local correlation structure is dominant in the target data. Furthermore, it can conveniently make use of biological constraints to get a better estimate. Experiments show that our algorithm can achieve a significant reduction of error compared existing algorithms.
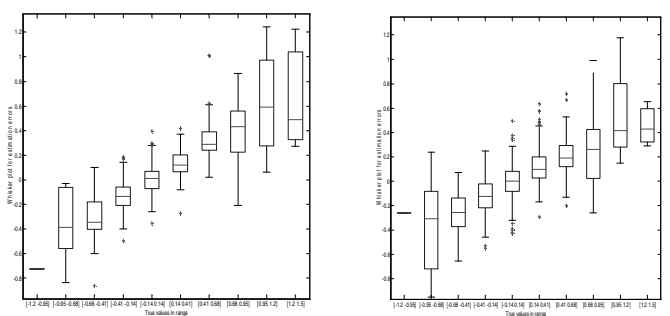

Fig. 4. Box and whisker plots of the estimation errors relative to the true values in range. (left) KNNimpute, and (bottom) POCSimpute.

Acknowledgement: This work is supported by a CityU interdisciplinary grant (project 9010003) and strategic research grant (project 7001706).

\section{REFERENCES}

[1] O. Troyanskaya, et al. "Missing values estimation methods for DNA microarrays," Bioinformatics, 17, 520525, 2001.

[2] X. C. Gan, A. W. C. Liew and Yan, H., "Missing value estimation for microarray data based on projection onto convex sets method," Int'l Conf. Pat. Rec. (ICPR2004), Cambridge, UK, III: 782-785, 2004..

[3] P. T. Spellman, P.T. et al., "Comprehensive Identification identification of cell cycle-regulated genes of the yeast Saccharomyces cerevisiae by microarray hybridization," Mol. Biol. Cell., 9, 32733297, 1998.

[4] Z. Bar-Joseph, et al., "Deconvolving cell cycle expression data with complementary information," Bioinformatics, 20, i23-i30, 2004.

[5] H. Stark and Y. Yang, Vector Space Projections, John Wiley\& Sons, New York, 1998.

[6] X. C. Gan, A. W. C. Liew and H. Yan, "Blocking artifact reduction in compressed images based on edge-adaptive quadrangle meshes," J. Visual Communications \& Representation, 14(4):492-507, 2003.

[7] B $\emptyset$, T.H. Dysvik, B. and Jonassen, I. LSimpute: accurate estimation of missing values in microarray data with least squares method. Nucleic Acids Res.,32, e34, 2004

[8] DeRisi,J.L., Iyer,V.R. and Brown,P.O. Exploring the metabolic and genetic control of gene expression on a genomic scale. Science, 278, 680-686, 1997. 